Med Klin Intensivmed Notfmed 2022 · 117:83-84 https://doi.org/10.1007/s00063-021-00894-1 Online publiziert: 10. Januar 2022

(c) The Author(s), under exclusive licence to Springer Medizin Verlag GmbH, ein Teil von Springer Nature 2021

\section{Erratum zu: Medikamentöse Therapie des Kreislaufschocks}

Reimer Riessen · Rubi Stephani Hellwege

Internistische Intensivstation, Department für Innere Medizin, Universitätsklinikum Tübingen, Tübingen, Deutschland

\section{Erratum zu:}

Med Klin Intensivmed Notfmed 2021

https://doi.org/10.1007/s00063-021-

00838-9

In der ursprünglichen Version des Artikels "Medikamentöse Therapie des Kreislaufschocks" ist in Tab. 3 bei der Substanz Vasopressin irrtümlich ein Bolus von $40 \mathrm{U}$ aufgelistet worden. Vasopressinboli in dieser Dosis sind in früheren Studien im Rahmen von kardiopulmonalen Reanimationen eingesetzt worden, ohne dass ein klarer positiver Effekt auf das Überleben gezeigt werden konnte. Im Rahmen der Behandlung des Kreislaufschocks sollten Vasopressinboli nicht eingesetzt werden, da ein hohes Risiko von Nebenwirkungen besteht, die durch eine schwere Vasokonstriktion ausgelöst werden können. Stattdessen kann beim Kreislaufschock erwogen werden, bei einem hohen Noradrenalinbedarf eine niedrigdosierte Vasopressindauerinfusion $(0,01-0,04 \mathrm{U} / \mathrm{min})$ zusätzlich zu verabreichen.

Der ursprüngliche Beitrag wurde korrigiert.

\section{Korrespondenzadresse}

\section{Prof. Dr. med. Reimer Riessen}

Internistische Intensivstation, Department für Innere Medizin, Universitätsklinikum Tübingen Otfried-Müller-Str. 10, 72076 Tübingen,

Deutschland

reimer.riessen@med.uni-tuebingen.de
Die Online-Version des Originalartikels ist unter https://doi.org/10.1007/s00063-021-00838-9 zufinden. 
Hier steht eine Anzeige.

黑 Springer 